\title{
Distinction des espèces de Siphomycètes par l'examen en microscopie électronique à balayage
}

par M. ANSEL et M. THIBAUT

Laboratoire de Parasitologie et Mycologie ( $\mathrm{P}^{\mathrm{r}} \mathrm{M}$, LARIVIÈRE)

U.E.R. Cordeliers, 15, rue de l'Ecole-de-Médecine, F 75006 Paris

\begin{abstract}
Résumé
Quelques espèces de Siphomycètes ont été examinées au microscope électronique à balayage. Cette nouvelle technique a permis d'apporter des renseignements taxinomiques.
\end{abstract}

\section{Summary}

Some species of Siphomycetes were examined using a scanning electron microscope. This new technique has provided taxinomic information.

L'examen des champignons par le microscope électronique à balayage, entraîne certes du fait de la préparation dans l'évaporateur de carbone, un certain affaissement des hyphes. Néanmoins, la plupart des organes caractéristiques de reproduction gardent leurs structures aussi bien conservées qu'après les fixations et colorations utilisées en microscopie optique. Mais l'examen en microscopie électronique à balayage permet de préciser et de distinguer des caractères spécifiques qui ne peuvent être vus avec le microscope photonique. Cet examen complémentaire si utile devient donc parfois indispensable.

A titre d'exemple, nous présentons les aspects de quelques Siphomycètes qui faciliteront désormais le diagnose d'espèces étroitement apparentées. 


\section{Matériel et techniques.}

Les espèces étudiées ont été les Rhizopus arhizus, Rhizopus equinus et Rhizopus nigricans.

Les échantillons des différentes espèces, préalablement fixés aux vapeurs de formol, sont transportés sur de petits cylindres en cuivre qui sont ensuite placés dans un évaporateur de carbone. Ils sont d'abord recouverts d'un mince film de carbone, puis reçoivent une fine projection d'or - palladium. Ils sont ensuite examinés au microscope électronique à balayage (JSM U3 Jeolco) travaillant à des tensions d'accélération comprises entre 15 et 25 kilovolts.

\section{Observations.}

L'extrémité du cystophore, contrairement à ce que l'on voit chez les Mucor, se termine chez les Rhizopus et d'autres genres en un évacement ou apophyse qui se continue à l'intérieur du sporange par la columelle. Vraisemblablement lors de la préparation dans l'évaporateur de carbone, la chaleur provoque la rétraction de cette apophyse et de l'extrémité du cystophore vers l'intérieur de la columelle. Les images mon-

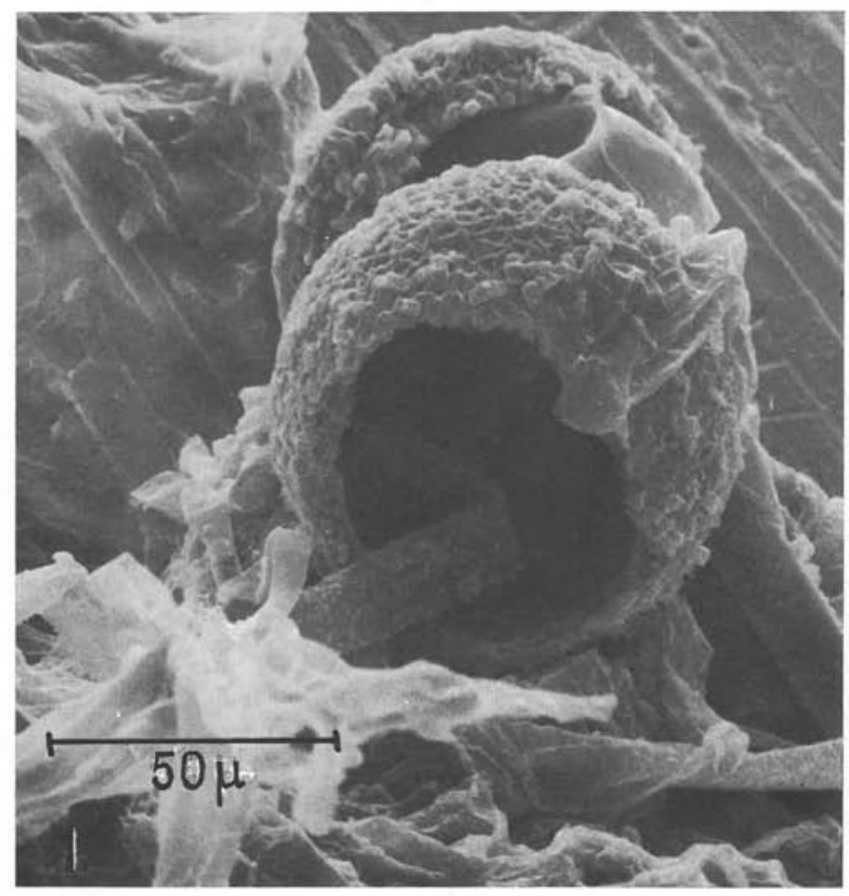

Fig. 1. - Rhizopus arhizus. Noter l'enfoncement à la base du sporange 
trent cette sorte d'enfoncement à la base du sporange et la formation d'une cavité (figure 1). On ne voit donc pas l'apophyse du cystophore supportant le sporocyste.

Chez le Rhizopus arhizus, le stipe paraît saupoudré de petits cristaux (fig. 2). La paroi du sporange est alvéolée (fig. 3-4). Chaque alvéole se présente sous forme d'une coupe indépendante comme pédicellée. L'intérieur des petites coupes du Rhizopus arhizus n'est pas une surface unie mais présente de longs plis traversant la coupe d'un bord à l'autre (fig. 5).

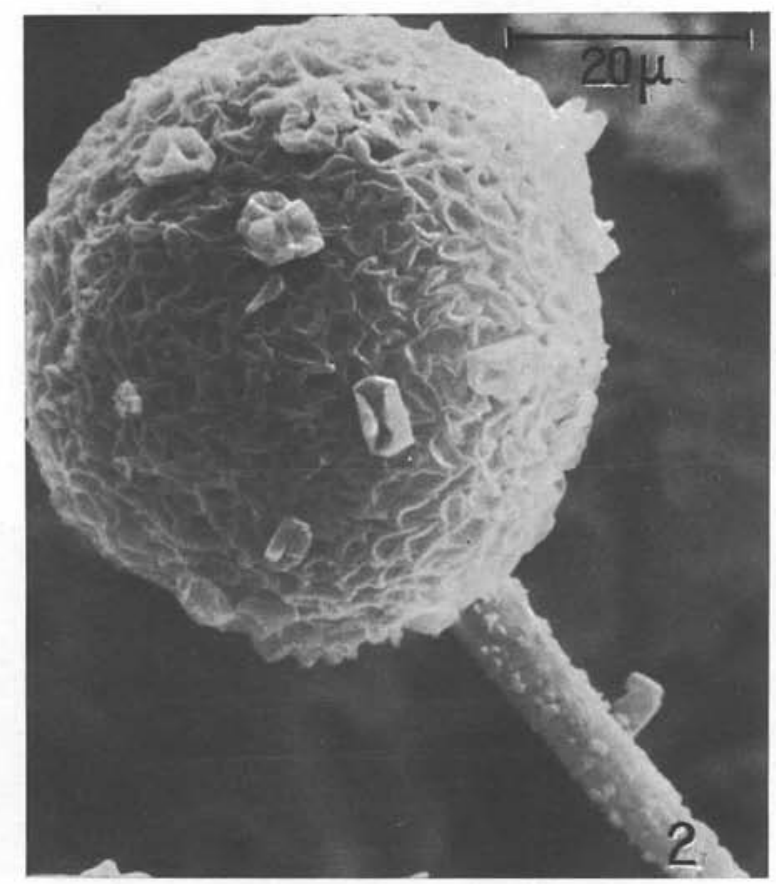

FIG. 2. - Rhizopus arhizus. Stipe paraissant saupoudré de petits cristaux

Chez le Rhizopus equinus, la microscopie à balayage montre l'aspect alvéolé de l'extérieur des membranes des sporanges (fig. 6). Ces membranes ont tout à fait l'aspect d'une tête de morille et les bords des alvéo'es forment une sorte de réseau continu (fig. 7-8). Les pourtours des alvéoles sont confluents. Constantin et Lucet, en décrivant l'espèce equinus avaient signalé l'observation facile de chlamydospores intercalaires. La microscopie à balayage permet de remarquer leur surface lisse et leur forme allongée (fig. 9).

Chez le Rhizopus nigricans, le stipe est lisse. Lors de la déhiscence du sporange, celui-ci se vide de ses spores et les parois de la columelle s'affaissent en se retournant 

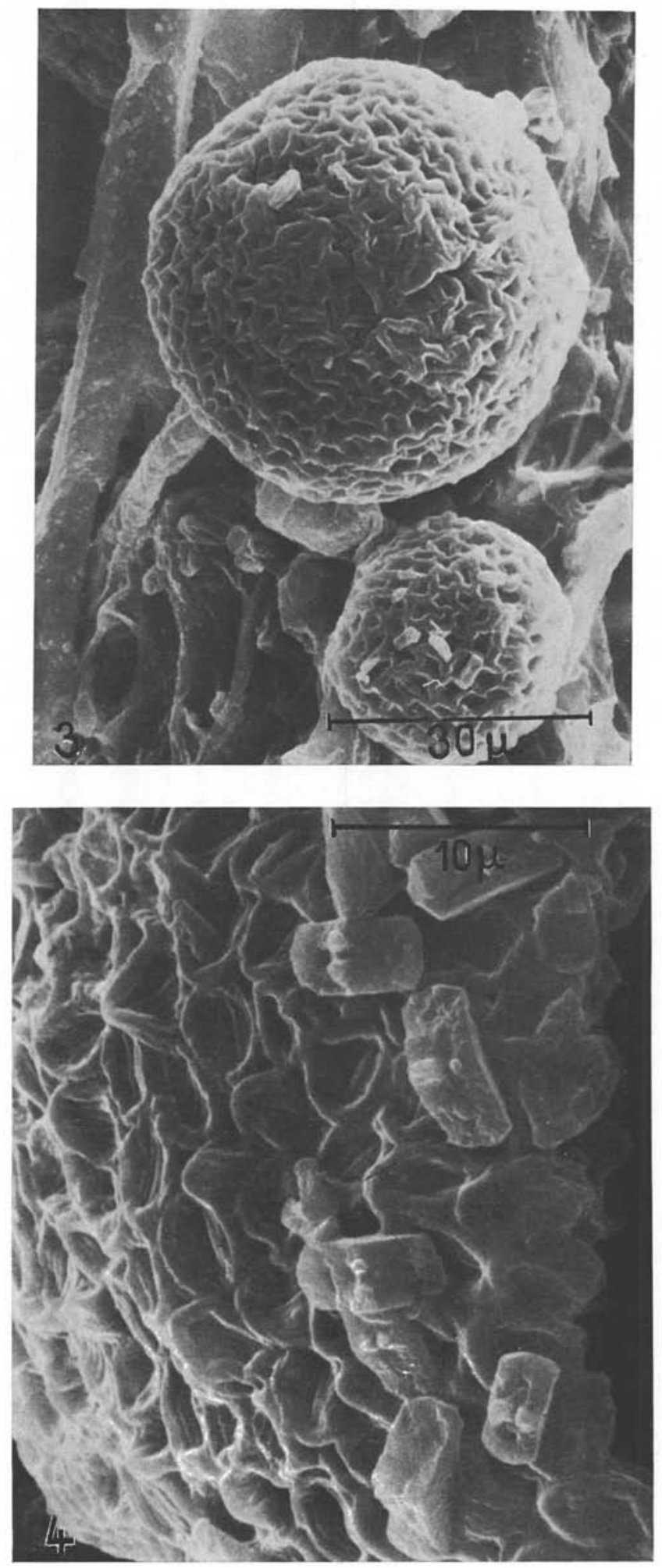


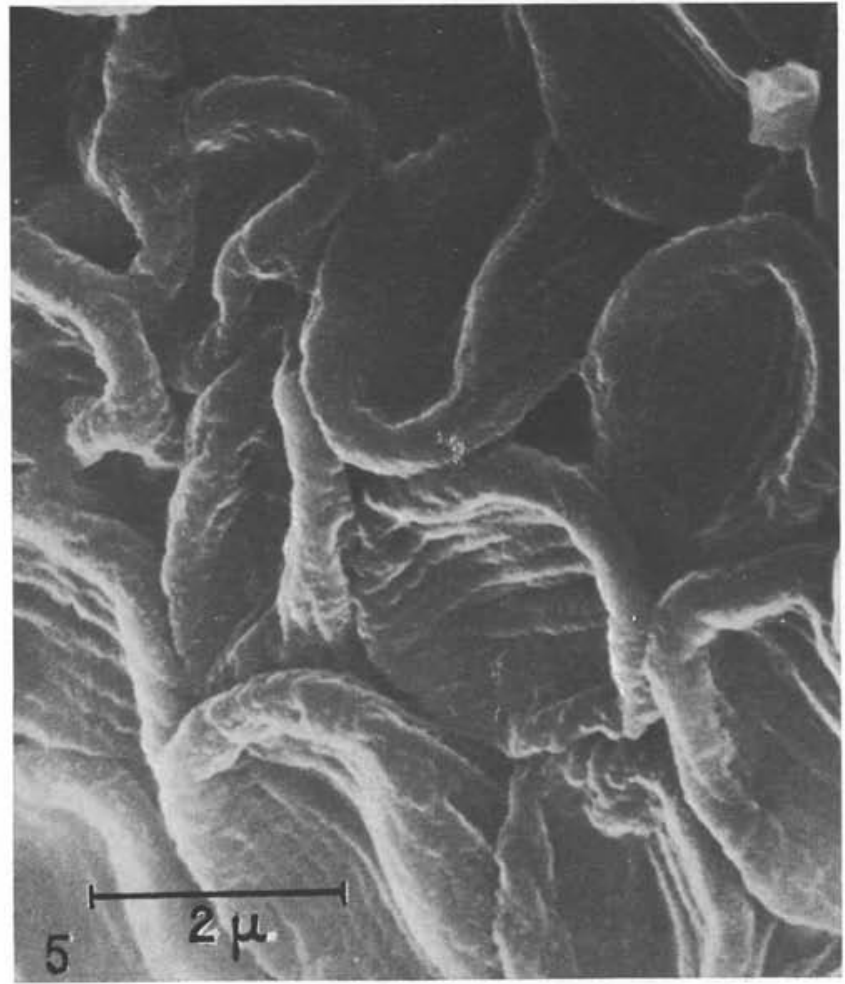

Fig. 5. - Rhizopus arhizus. Détail de la photo précédente

Fig. 3. - Rhizopus arhizus. Remarquer l'aspect alvéolé de la paroi du sporange

FIg. 4. - Rhizopus arhizus. Structure alvéolée de la paroi du sporange 

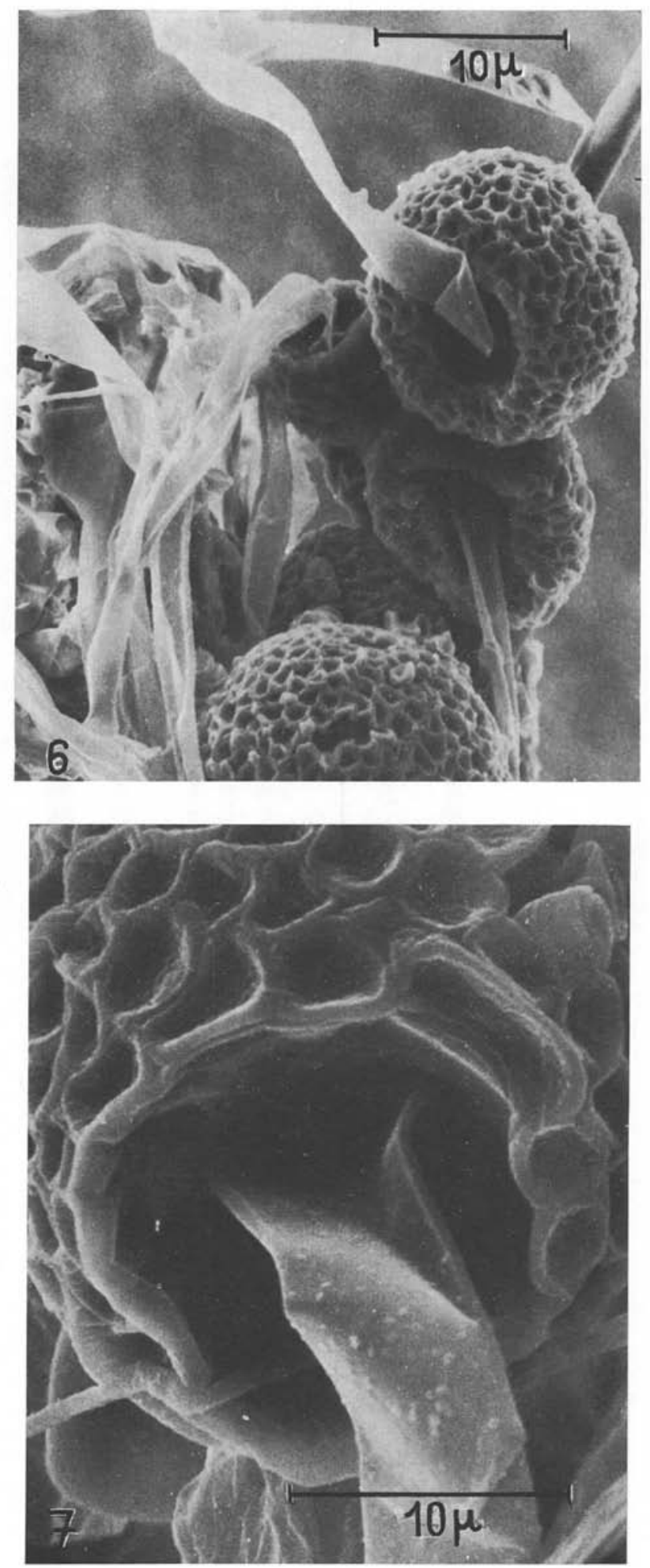


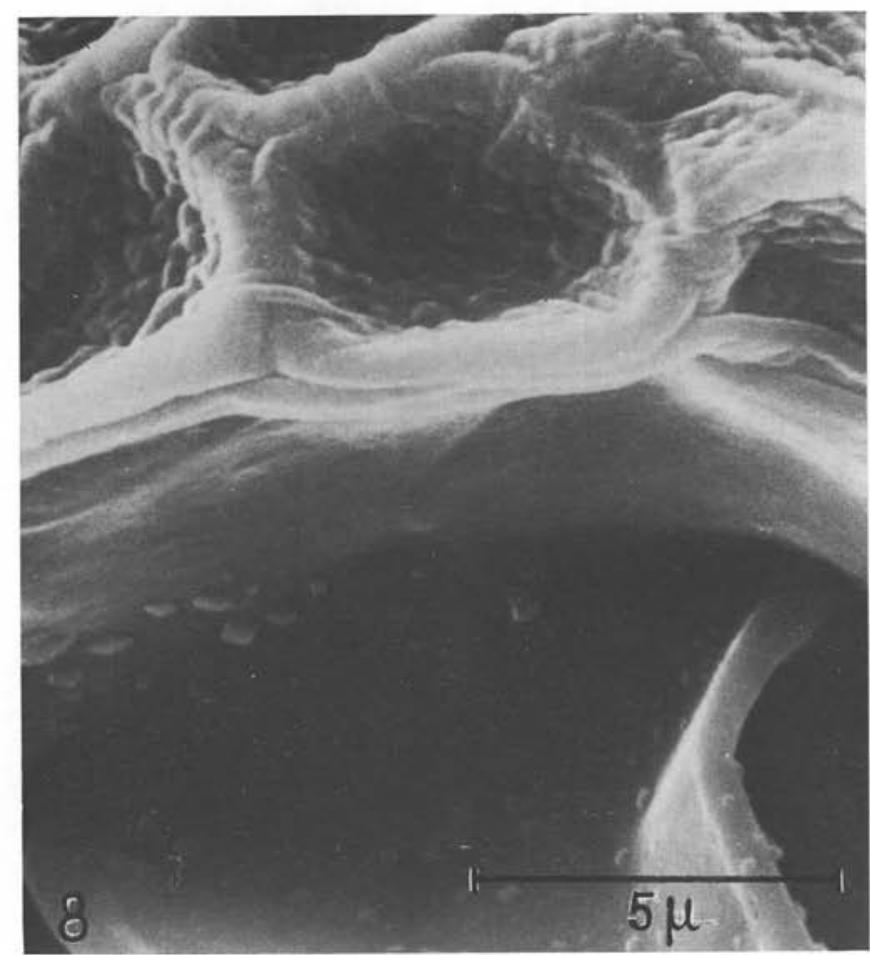

FIG. 8. - Sporange du Rhizopus equinus. Détail de la photo précédente

FIG. 6. - Sporanges du Rhizopus equinus

Fig. 7. - Vue inférieure d'un sporange du Rhizopus equinus. Noter les alvéoles de la paroi externe 

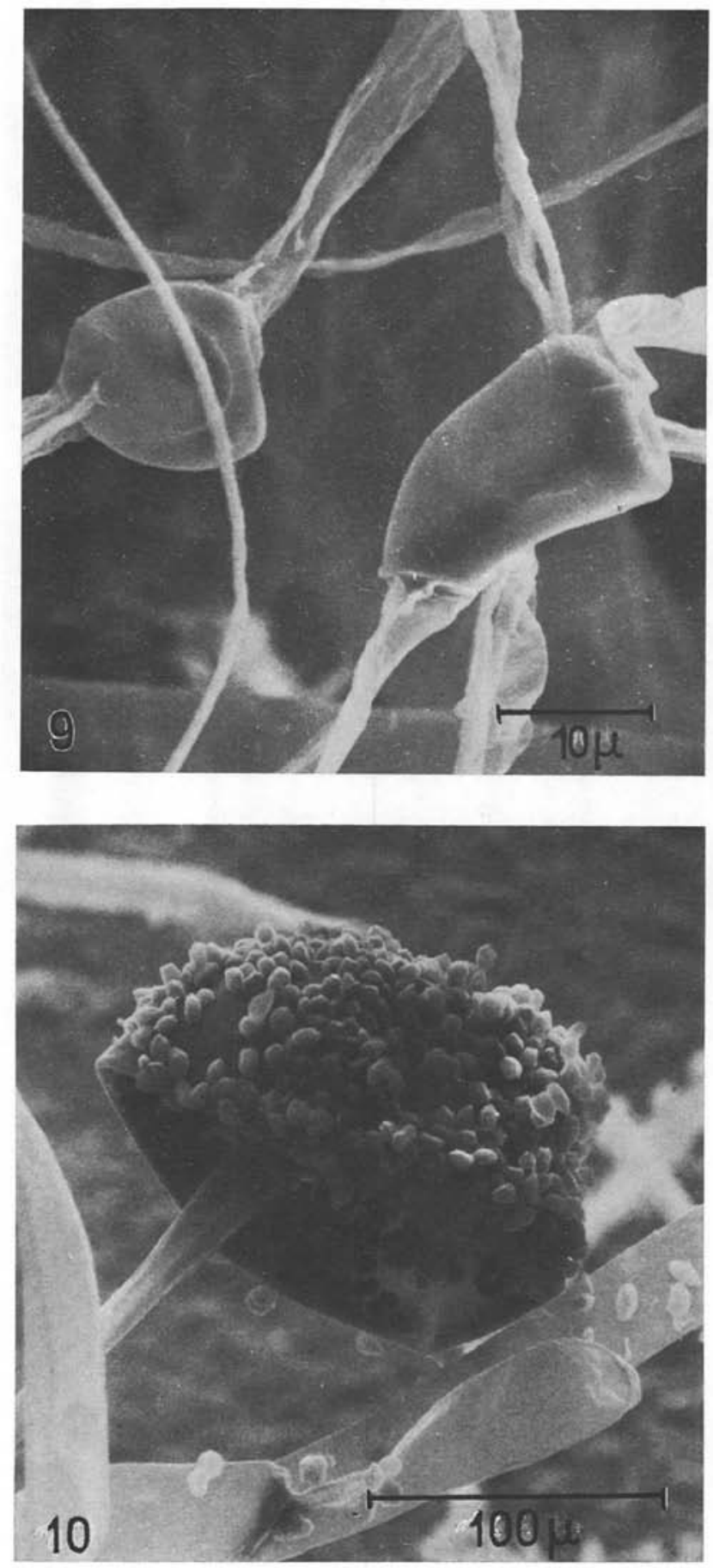


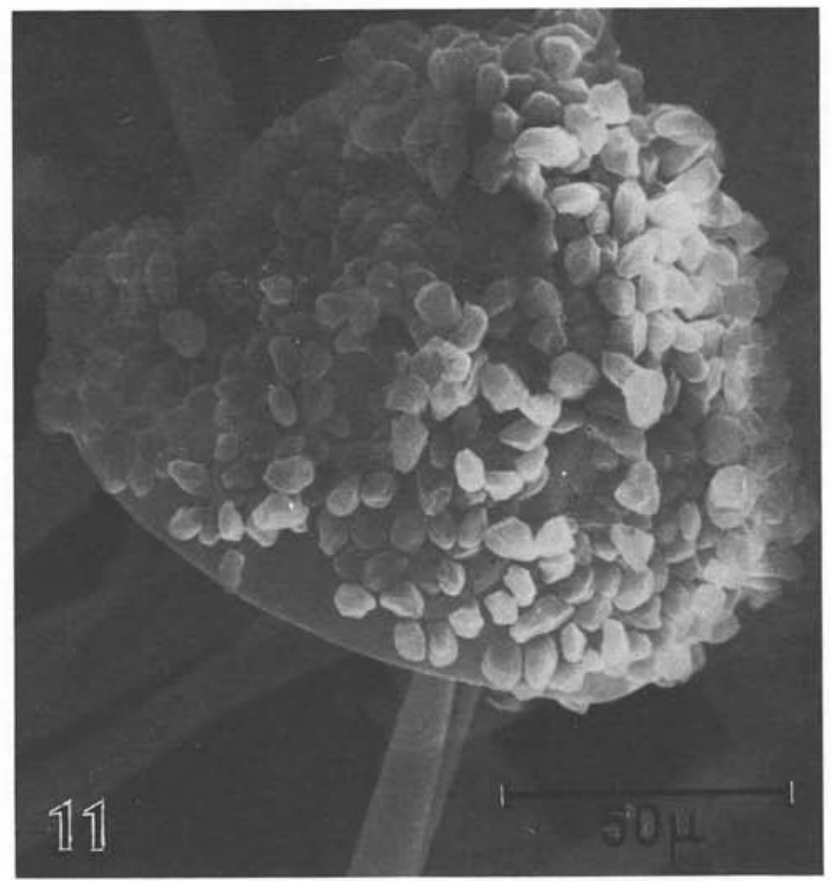

FIG. 11. - Rhizopus nigricans, sporange dehiscent

FIG. 9. - Chlamydospores du Rhizopus equinus. Noter leur aspect absolument lisse

FIG. 10. - Rhizopus nigricans, sporange dehiscent 
(fig. 10-11). De nombreuses spores restent adhérentes à la surface lisse de la columelle. Les spores sont côtelées comme du velours, les côtes dispozées dans le sens de la longueur de la spore étant cependant flexueuses (fig. 12). Toutes les côtes semblent aboutir aux pôles de la spore, mais la microscopie à balayage ne permet pas d'y voir un pore germinatif.

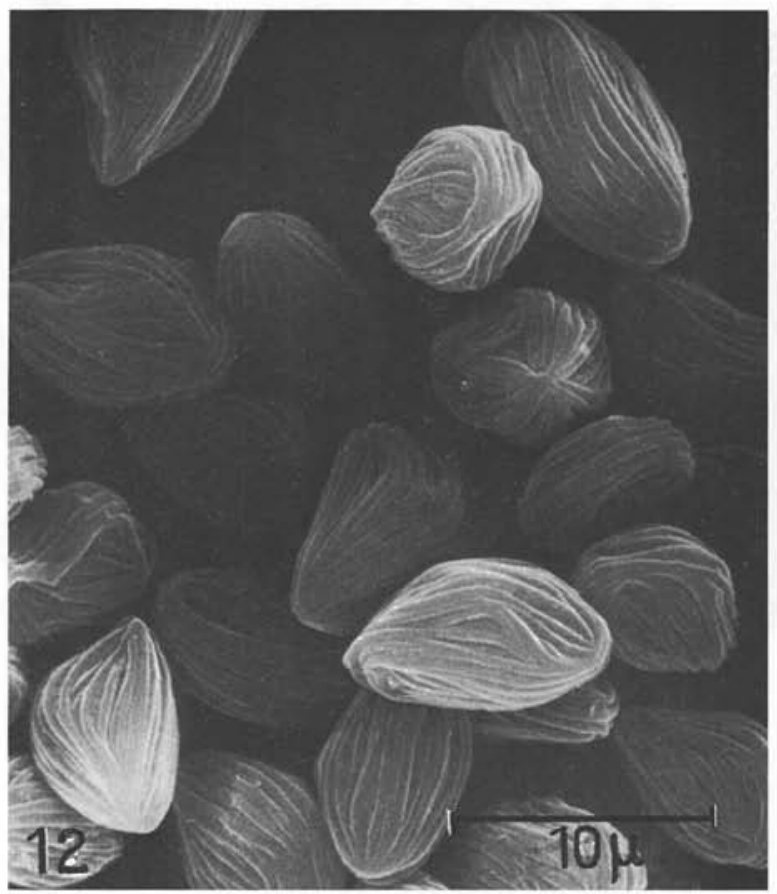

Fig. 12. - Spores du Rhizopus nigricans. Remarquer leur aspect côtelé

\section{Conclusion.}

La microscopie à balayage permet d'observer chez les champignons Siphomycètes des structures caractéristiques qui facilitent la distinction d'espèces voisines par simple examen idu revêtement des sporophores et de l'aspect des sporanges. D'autre part, le microscope à balayage permet seul de faire la distinction entre les alvéoles des Rhizopus arhizus et equinus. Il faut en effet des grandissements de 5 à 10000 pour voir ces différences. Enfin, l'ornementation si particulière de la cuticule des spores peut être précisée d'une façon particulièrement nette. 


\section{Bibliographie}

Bernard (P.-N.), 1914. - Sur un Rhizopus pathogène de l'homme. Bull. Soc. Mycol. France, 30, 230-232.

Costantin et Lucet, 1903. - Sur un Rhizopus pathogène. Bull. Soc. Mycol. France, 29, 200-216.

Thibaut (M.) et Ansel (M.), 1971. - Essai d'utilisation du microscope électronique à balayage pour l'étude morphologique des champignons. Ann. de Parasitol. hum. et comp., 46, 103-107.

Van Tieghem (Ph.), 1875. - Nouvelles recherches sur les Mucorinées. Ann. Sc. Nat., 6, 5-175,

Vuillemin (P.), 1903. - Importance taxinomique de l'appareil zygosporé des Mucorinées. Bull. Soc. Mycol. France, 29, 106-118.

—, 1931. - Les champignons parasites et les mycoses de l'homme, Lechevalier éd., Paris.

Tous droits de traduction, d'adaptation et de reproduction par tous procédés réservés pour tous pays

La loi du 11 mars 1957 n'autorisant, aux termes des alinéas 2 et 3 de l'article 41, d'une part, que les «copies ou reproductions strictement réservées à l'usage privé du copiste et non destinées à une utilisation collective» et, d'autre part, que les analyses et les courtes citations dans un but d'exemple et d'illustration, «toute représentation ou reproduction intégrale ou partielle, faite sans le consentement de l'auteur ou de ses ayants-droit ou ayants-cause, est illicite s (alinéa $1^{\text {er de }}$ l'article 40).

Cette représentation ou reproduction, par quelque procédé que ce soit, constituerait donc une contrefaçon sanctionnée par les articles 425 et suivants du Code pénal.

(C) 1973, Masson et $C^{i e}$, Paris 